\title{
Investigation of the stator inductances of the expanded Park model and an approach on parameter identification using the evolution strategy
}

\author{
Christoph Schmuelling ${ }^{1}$, Christian Kreischer ${ }^{1}$, Marek Golebiowski ${ }^{2}$ \\ ${ }^{1} T U$ Dortmund University \\ Chair of Energy Conversion \\ Emil-Figge-Str. 70, 44227 Dortmund, Germany \\ e-mail: christoph.schmuelling@tu-dortmund.de \\ ${ }^{2}$ Rzeszow University of Technology \\ Faculty of Electrical and Computer Engineering \\ ul. Wincentego Pola 2, 35-959 Rzeszów, Poland \\ e-mail:yegolebi@prz.edu.pl
}

(Received: 17.08.15, revised: 21.03.2016)

\begin{abstract}
Commonly, the Park model is used to calculate transients or steady-state operations of synchronous machines. The expanded Park theory derives the Park equations from the phase-domain model of the synchronous machine by the use of transformations. Thereby, several hypothesis are made, which are under investigation in this article in respect to the main inductances of two different types of synchronous machines. It is shown, that the derivation of the Park equations from the phase-domain model does not lead to constant inductances, as it is usually assumed for these equations. Nevertheless the Park model is the most common analytic model of synchronous machines. Therefore, in the second part of this article a method using the evolution strategy is shown to obtain the parameters of the Park model.
\end{abstract}

Key words: evolution strategy, FEM, Park equations, Park theory, synchronous machines, transients

\section{Introduction}

Due to the growth of renewable energy and, as a consequence thereof, fluctuating energy feed, new concepts for energy systems have to be developed. For instance, there are new control strategies for variable speed wind turbines [1] or the use of induction machines as a brushless excitation system of turbo generators [2]. In the wake of the volatile energy feed, more and more transients occur in the grid. Hence, it is necessary to have good analytic models of synchronous machines. The Park equations [3] are commonly used as an analytical model for 
calculations. But it is difficult to determine the parameters for this model that are necessary for good accuracy. In the expanded Park theory, e.g. in [4] and [5], the equations of the phase-domain model are transformed to the Park equations. Hence, the parameters of the phase-domain model yield directly to the parameters that are needed for the Park equations. In this article it is shown that the expanded Park theory depends on assumptions which result in incorrect Park inductances when deriving them from the phase-domain inductances. These vague inductances lead to inaccurate simulation results. Especially, improper stator currents are critical, as the electromagnetic forces of the machine depend quadratic of them.

As the Park model is the common analytic model of the synchronous machine, an advanced method to determine the parameters of the Park model is shown in the second part of this article. This method uses measurements of synchronous machines or the results of finite element analysis in combination with the evolution strategy.

\section{Expanded Park theory of synchronous machines}

The Park model is based on the transformation of the time- and rotor angle-dependent parameters and values to a rotor fixed coordinate system. The main benefit is that the inductances have constant values and the mathematical system is decoupled. In the expanded Park theory, the equations of the phase-domain model are transformed to the Park equations. As a result of the transformation, all parameters of the Park equations are deduced from the parameters of the phase-domain model.

The equations of the phase-domain model can be derived directly from the energies stored in the system of the synchronous machine using the Euler-Lagrange equation:

$$
\frac{\partial H}{\partial q_{k}}-\frac{\mathrm{d}}{\mathrm{d} t} \frac{\partial H}{\partial \dot{q}_{k}}=0,
$$

where $H$ is the Lagrangian and $q_{k}$ is the $k$-th generalized coordinate. The Lagrangian $H$ of the considered system consists of the kinetic energy, the potential energy, the losses and the input energy of the machine [4].

The generalized coordinates of a synchronous machine are the mechanical rotor angle $\varphi$ and the electric charges $Q$ of each winding. Usually there are three stator windings, one field winding and two surrogate damper windings, hence the number of the generalized coordinates is seven. All energies of the synchronous machine can be found in literature, for instance, in [4] and [6].

The result of the Euler-Lagrange equation (1) are six voltage equations

$$
\left[\begin{array}{c}
V_{\mathrm{S}} \\
V_{\mathrm{R}}
\end{array}\right]=\left[\begin{array}{cc}
R_{\mathrm{S}} & 0 \\
0 & R_{\mathrm{R}}
\end{array}\right] \cdot\left[\begin{array}{c}
I_{\mathrm{S}} \\
I_{\mathrm{R}}
\end{array}\right]+\frac{\mathrm{d}}{\mathrm{d} t}\left(\left[\begin{array}{cc}
M_{\mathrm{SS}} & M_{\mathrm{SR}} \\
M_{\mathrm{RS}} & M_{\mathrm{RR}}
\end{array}\right] \cdot\left[\begin{array}{c}
I_{\mathrm{S}} \\
I_{\mathrm{R}}
\end{array}\right]\right),
$$

and one mechanical equation 


$$
\frac{\mathrm{d}}{\mathrm{d} t} J \dot{\varphi}+D_{R} \frac{\mathrm{d} \varphi}{\mathrm{d} t}=\frac{1}{2} \sum_{k=1}^{6} \sum_{n=1}^{6} \frac{\partial M_{k, n}}{\partial \varphi} I_{k} I_{n}+m_{a}
$$

that describe the synchronous machine. $J$ is the moment of inertia, $D_{R}$ is the damping constant of the rotor shaft and $m_{a}$ is the external torque. The inductance matrices $\boldsymbol{M}$ are full matrices and the elements of $\boldsymbol{M}_{\mathbf{S S}}, \boldsymbol{M}_{\mathbf{S R}}$ and $\boldsymbol{M}_{\mathbf{R S}}$ are dependent on the rotor angle $\varphi$ :

$$
\begin{gathered}
\boldsymbol{M}_{\mathbf{S S}}=\left[\begin{array}{lll}
L_{a a}(\varphi) & L_{a b}(\varphi) & L_{a c}(\varphi) \\
L_{b a}(\varphi) & L_{b b}(\varphi) & L_{b c}(\varphi) \\
L_{c a}(\varphi) & L_{c b}(\varphi) & L_{c c}(\varphi)
\end{array}\right], \\
\boldsymbol{M}_{\mathbf{S R}}=\left[\begin{array}{lll}
L_{a f}(\varphi) & L_{a d}(\varphi) & L_{a q}(\varphi) \\
L_{b f}(\varphi) & L_{b d}(\varphi) & L_{b q}(\varphi) \\
L_{c f}(\varphi) & L_{c d}(\varphi) & L_{c q}(\varphi)
\end{array}\right], \\
\boldsymbol{M}_{\mathbf{S R}}=\boldsymbol{M}_{\mathbf{R S}}{ }^{\mathrm{T}},
\end{gathered}
$$

where the subscripts $a, b$ and $c$ represent the stator windings, $f$ the field winding, $d$ and $q$ the damper windings in the direct $(d)$ and quadrature $(q)$ axis.

The self-inductances in $\boldsymbol{M}_{\text {SS }}$ (4) of a two pole synchronous machine are calculated by

$$
\begin{gathered}
L_{a a}(\varphi)=L_{\sigma}+M_{1}+M_{2} \cdot \cos (2 \varphi), \\
L_{b b}(\varphi)=L_{\sigma}+M_{1}+M_{2} \cdot \cos \left(2 \cdot\left(\varphi-\frac{2 \pi}{3}\right)\right), \\
L_{c c}(\varphi)=L_{\sigma}+M_{1}+M_{2} \cdot \cos \left(2 \cdot\left(\varphi+\frac{2 \pi}{3}\right)\right),
\end{gathered}
$$

and the mutual inductances, for example $L_{c a}(\varphi)$

$$
L_{c a}(\varphi)=-\frac{M_{1}}{2}+M_{2} \cdot \cos \left(2 \cdot\left(\varphi-\frac{2 \pi}{3}\right)\right) .
$$

The self-inductances consist of a steady component $\left(L_{\sigma}+M_{1}\right)$ and an alternating component $\left(M_{2}\right)$. In Fig. 1, the phase-dependent course of the self-inductance $L_{a a}$ is depicted. Also the mutual inductances consist of a steady component $\left(-M_{1} / 2\right)$ and an alternating component $\left(M_{2}\right)$.

The hypothesis of the expanded Park theory is, that the alternating components of self and mutual inductances are equal. Furthermore, the magnitudes of the steady components of the self-inductances minus the leakage inductance $L_{\sigma}$ are twice as big as the magnitudes of the steady components of the mutual inductances [5]. 
To derive Park equations from the phase-domain model, additional hypothesis have to be made:

- linear magnetic material,

- no eddy currents,

- a sinusoidal distribution of the stator current,

- symmetrical stator.

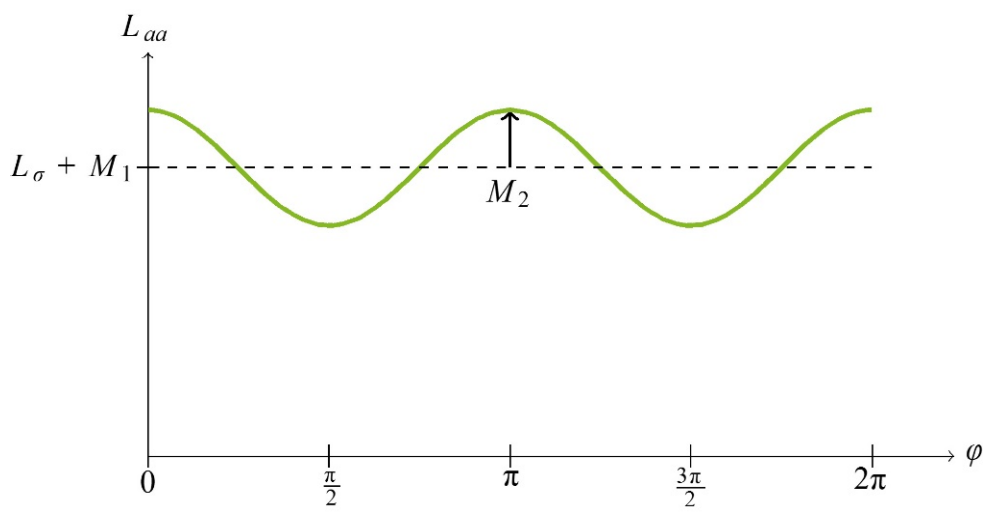

Fig. 1. Phase-dependent course of $L_{a a}$

With a transformation matrix [6] the equations of the phase-domain model are transformed to the Park equations with constant inductances. After transformation, the voltage equations of the direct and quadrature axis are:

$$
V_{d}=R_{s} I_{d}+\frac{\mathrm{d}}{\mathrm{d} t}\left[L_{d} I_{d}+L_{m d}\left(I_{f}^{\prime}+I_{d d}^{\prime}\right)\right]-\dot{\varphi}\left(L_{q} I_{q}+L_{m q} I_{d q}^{\prime}\right)
$$

and

$$
V_{q}=R_{s} I_{q}+\frac{\mathrm{d}}{\mathrm{d} t}\left[L_{q} I_{q}+L_{m q} I_{d q}^{\prime}\right]-\dot{\varphi}\left(L_{d} I_{m d}+L_{m d}\left(I_{f}^{\prime}+I_{d q}^{\prime}\right)\right)
$$

with

$$
L_{d}=L_{m d}+L_{\sigma} \text { and } L_{q}=L_{m q}+L_{\sigma}
$$

the leakage inductance $L_{\sigma}$ and the mutual inductances $L_{m d}$ and $L_{m q}$. The subscript $d$ means parameters of the direct axis and $q$ of the quadrature axis. All rotor variables are referred to the stator [5]. $L_{m d}$ and $L_{m q}$ can be calculated directly from the inductances of the phase-domain model:

$$
L_{m d}=\frac{3}{2}\left(M_{1}+M_{2}\right),
$$




$$
L_{m q}=\frac{3}{2}\left(M_{1}+M_{2}\right) .
$$

The Equations (9), (10) and the equations of the rotor circuits [5] result in the equivalent circuit diagrams shown in Figs. 2 and 3. After transformation the mechanical Equation (3) becomes

$$
\frac{\mathrm{d}}{\mathrm{d} t} J \dot{\varphi}+D_{R} \frac{\mathrm{d} \varphi}{\mathrm{d} t}=\frac{3}{2}\left(\Psi_{d} I_{q}-\Psi_{q} I_{d}\right)+m_{a}
$$

with

$$
\Psi_{d}=L_{d} I_{d}+L_{m d}\left(I_{f}^{\prime}+I_{d d}^{\prime}\right) \text { and } \Psi_{q}=L_{q} I_{q}+L_{m q} I_{d q}^{\prime}
$$

$I_{d d}^{\prime}$ and $I_{d q}^{\prime}$ represent the stator referred currents of the damper windings in the $d$-respectively $q$-axis.

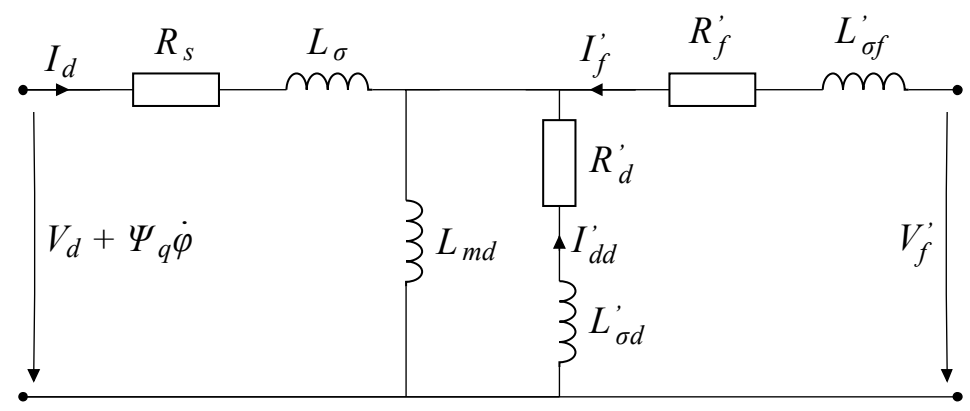

Fig. 2. Park $d$-axis circuit

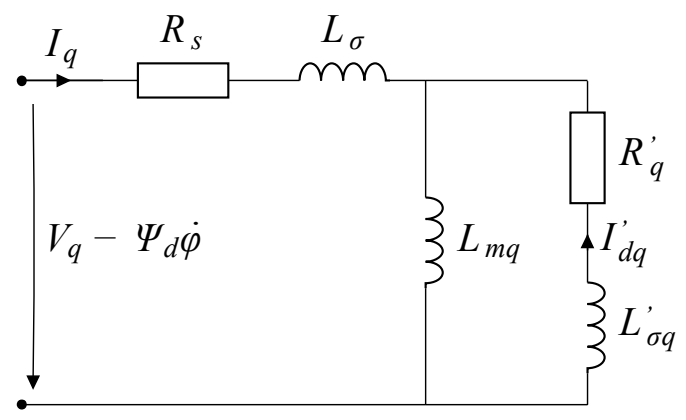

Fig. 3. Park $q$-axis circuit

In [14] it is shown that an additional inductance $L_{c d}^{\prime}$ has to be integrated in the voltage equations of the direct axis to calculate correct rotor currents (see Fig. 4). An extra inductance $L_{c q}^{\prime}$ has to be added in the voltage equations of the quadrature axis also if there are two damper circuits. In this article, there is only one damper circuit in each axis, thus $L_{c q}^{\prime}$ can be neglected. 


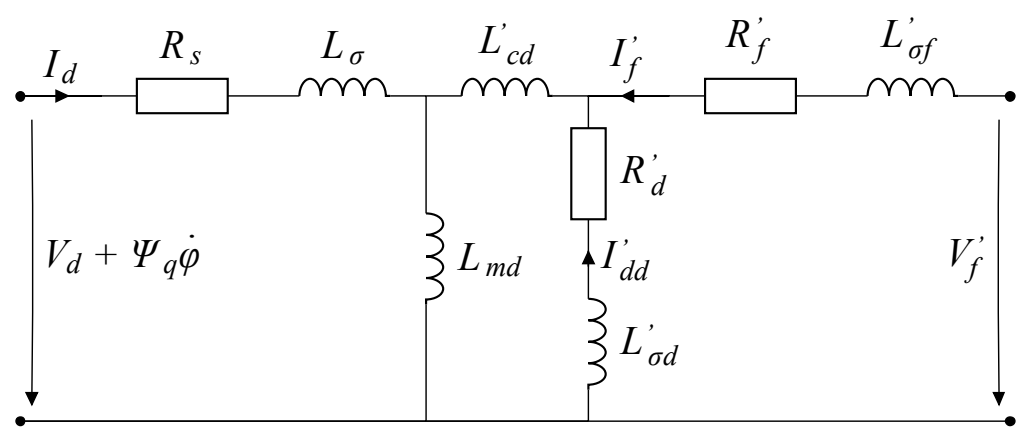

Fig. 4. Park $d$-axis circuit with additional inductance $L_{c d}^{\prime}$

\section{Analysis of inductances $L_{m d}$ and $L_{m q}$}

As it results from chapter 2 the mutual Park inductances $L_{m d}$ and $L_{m q}$ are derived from the stator self and mutual inductances of the phase-domain model. In this chapter a closer look at these inductances is made. For that reason the inductances of two synchronous machines are under investigation. Thereby the classical hypothesis of no saturation, no eddy currents and a sinusoidal magnetomotive force ( $\mathrm{mmf}$ ) in the air gap are regarded. Since a real machine with stator slots has no sinusoidal distribution of the stator current, a fast Fourier transform (FFT) is applied to the inductance waveforms to extract the steady components and the first harmonics, which are adequate to the alternating components of the inductances (7) and (8). The first machine is a round rotor synchronous machine with a rated power of $50 \mathrm{~kW}$ (Fig. $5 \mathrm{a}$ ). The second machine has a salient pole rotor (Fig. 5b) and a rated power of $75 \mathrm{~kW}$. So the focus lies on two different types of synchronous machines to get a comprehensive result. Both machines are modeled with the finite element (FE) software Ansys Maxwell 2D. The FE model of the round rotor machine is verified with measurements. To consider the influences of the saturation, the machines are modeled with linear as well as with nonlinear magnetic material.

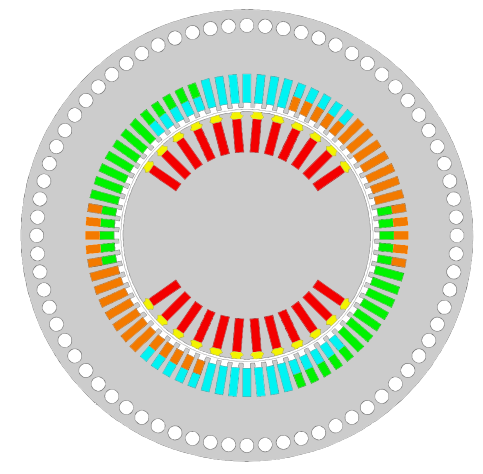

(a) round rotor

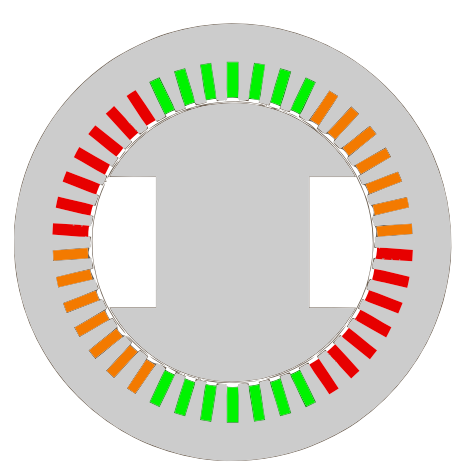

(b) salient pole rotor

Fig. 5. 2D cross section of the synchronous machines 
To determine the inductances of the machines with linear magnetic material, a direct current is injected in only one winding and the magnetic flux is evaluated in each winding. For instance, the self-inductance of stator winding $a$ and the mutual inductance between winding $a$ and $c$ are calculated by:

$$
\begin{aligned}
& L_{a a}=\frac{\Psi_{a}}{I_{a}}, \\
& L_{c a}=\frac{\Psi_{c}}{I_{a}} .
\end{aligned}
$$

$\Psi_{a}$ is the magnetic flux that is linked with stator winding $a$ and $I_{a}$ is the direct current of stator winding $a$. To consider saturation of the magnetic material, the currents of the operation points of interest have to be injected in all windings. Afterwards the distribution of the permeability is stored and the determination of the inductances is equivalent to the procedure with linear magnetic material. In this article only the static inductances are under investigation.

The stator self and mutual inductances of both machines without regarding saturation are depicted in Figs. 6 and 7. Based on a symmetrical stator geometry it is sufficient to consider just one stator self-inductance and one stator mutual inductance. The other inductances distinguish in another angle but not in the alternating or in the steady component.

\subsection{Round rotor synchronous machine}

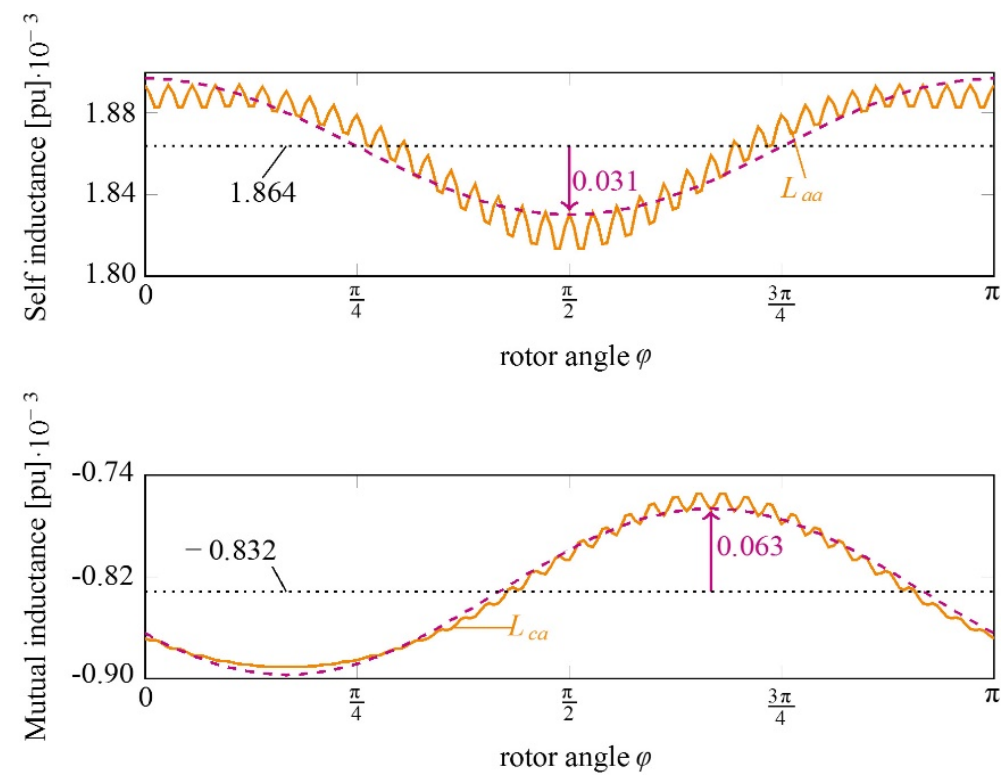

Fig. 6. Inductances of the round rotor machine and their fundamental waves (purple line) with linear magnetic material 
The self-inductance of the stator $L_{a a}$ results in

$$
L_{a a}=1.864 \cdot 10^{-3}+3.13 \cdot 10^{-5} \cdot \cos (2 \varphi)[\mathrm{pu}],
$$

and the mutual inductance in

$$
L_{a a}=-0.832 \cdot 10^{-3}+6.25 \cdot 10^{-5} \cdot \cos \left(2\left(\varphi-\frac{2 \pi}{3}\right)\right)[\mathrm{pu}] .
$$

Following the results of the inductances, it is clearly discernible that the hypothesis of an equal amplitude of self and mutual inductance of the expanded Park theory is incorrect. The alternating component of the mutual inductance is twice the component of the self-inductance. Even the conjecture of the steady components is inaccurate. The leakage inductance, calculated by a FE method described in [7], is $L_{\sigma}=1.85 \cdot 10^{-5} \mathrm{pu}$ which yields

$$
1.864 \cdot 10^{-3}-1.85 \cdot 10^{-5} \neq(-2) \cdot\left(-0.832 \cdot 10^{-3}\right) \text {. }
$$

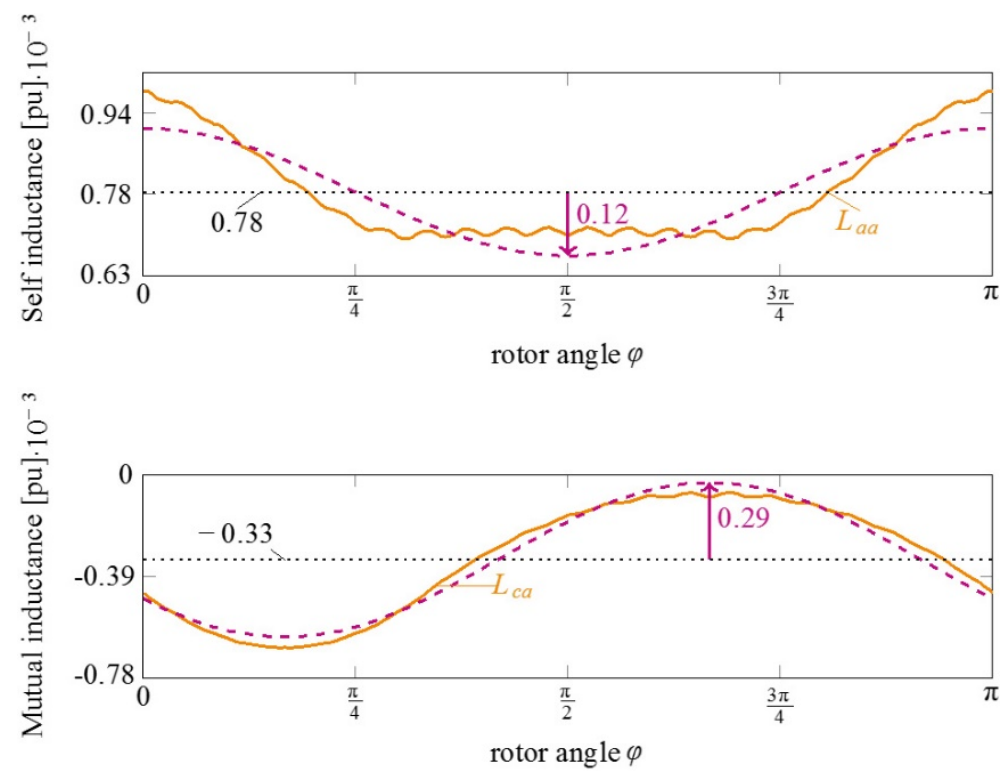

Fig. 7. Inductances of the salient pole rotor machine and their fundamental waves (purple line) with linear magnetic material

\subsection{Salient pole rotor synchronous machine}

The salient pole rotor machine shows the same results as the round rotor machine. The inductances are

$$
L_{a a}=0.78 \cdot 10^{-3}+1.2 \cdot 10^{-4} \cdot \cos (2 \varphi)[\mathrm{pu}],
$$




$$
\begin{gathered}
L_{a a}=-0.33 \cdot 10^{-3}+2.9 \cdot 10^{-4} \cdot \cos \left(2\left(\varphi-\frac{2 \pi}{3}\right)\right)[\mathrm{pu}], \\
L_{\sigma}=9.26 \cdot 10^{-6}[\mathrm{pu}] .
\end{gathered}
$$

According to the more unsymmetrical rotor geometry the alternating components of the inductances are larger than for the round rotor machine. But again the assumption of the same alternating components of stator self and mutual inductance is not correct.

\subsection{Saturation}

To investigate the influence of saturation on the inductances, the round rotor synchronous machine is modelled with nonlinear magnetic material. The inductances are calculated at no load operation with rated voltage. At this operation point, the material of the machine is highly saturated. The inductance waveforms are depicted in Fig. 8. Due to the high saturation of the direct axis all inductances have a phase shift of $\pi / 2 \mathrm{rad}$, a smaller steady component and a growing alternating component in comparison to the inductances of the synchronous machine with linear magnetic material. However, the inductances reveal the same behavior with and without saturation. The values of the components of self and mutual inductance are not equal.

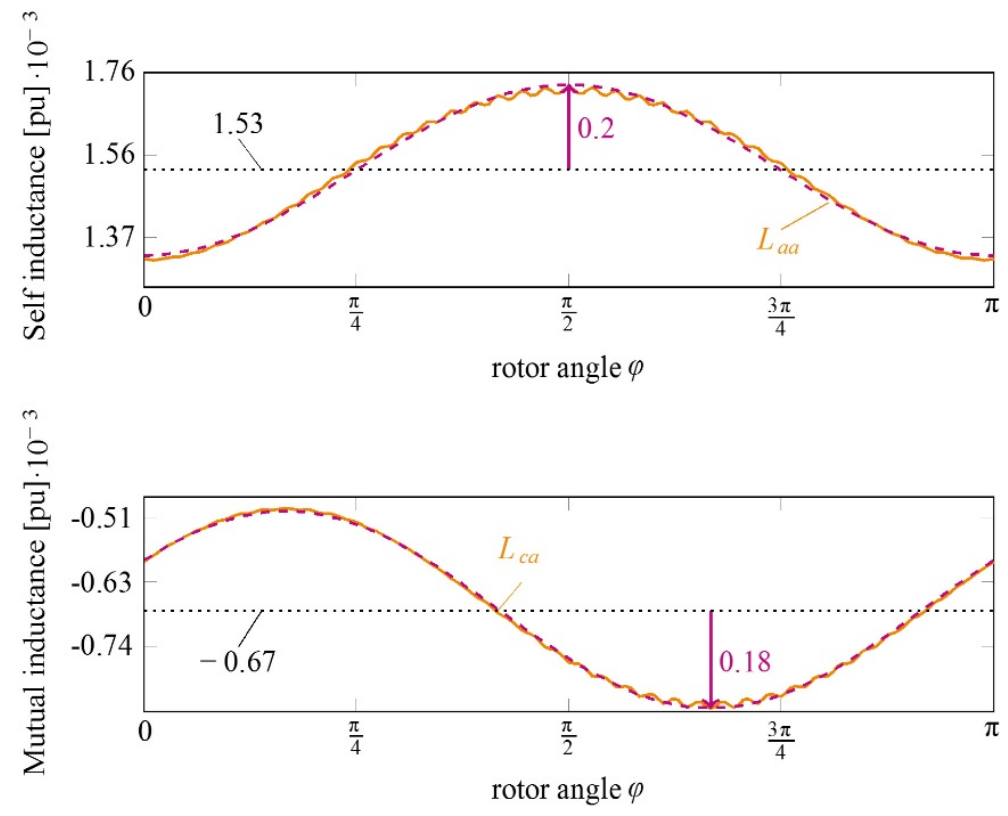

Fig. 8 Inductances of the round rotor machine and their fundamental waves (purple line) with nonlinear magnetic material

\subsection{New calculation of Park inductances}

According to the results of chapters 3.1 to 3.3 the inductances of Equations (7) and (8) have to be restated: 


$$
\begin{gathered}
L_{a a}(\varphi)=L_{\sigma}+M_{1}+M_{2} \cdot \cos (2 \varphi), \\
L_{c a}(\varphi)=\frac{M_{1}^{\prime}}{2}+M_{2}^{\prime} \cdot \cos \left(2\left(\varphi-\frac{2 \pi}{3}\right)\right),
\end{gathered}
$$

with $M_{1} \neq M^{\prime}{ }_{1}$ and $M_{2} \neq M^{\prime}{ }_{2}$.

These inductances are just valid without considering saturation. With the new stator self and mutual inductances the Park transformation does not lead to a decoupled system. The stator inductances matrix of the transformed system is:

$$
\left[\begin{array}{ccc}
L_{0}^{\prime} & \frac{M_{d q}}{2} \cos (3 \varphi) & -\frac{M_{d q}}{2} \sin (3 \varphi) \\
\frac{M_{d q}}{2} \cos (3 \varphi) & L_{d}^{\prime} & 0 \\
-\frac{M_{d q}}{2} \sin (3 \varphi) & 0 & L_{q}^{\prime}
\end{array}\right]
$$

where

$$
\begin{aligned}
& L_{d}^{\prime}=L_{\sigma}+M_{1}+M_{2}+\frac{M_{1}^{\prime}+M_{2}^{\prime}}{2} \quad L_{0}^{\prime}=L_{\sigma}+M_{1}-M_{1}^{\prime}, \\
& L_{q}^{\prime}=L_{\sigma}+M_{1}-M_{2}+\frac{M_{1}^{\prime}-M_{2}^{\prime}}{2} \quad M_{d q}=M_{2}-M_{2}^{\prime} .
\end{aligned}
$$

It can be seen, that some elements of the new stator inductances matrix (21) are a function of the rotor angle $\varphi$ and thereby a function of time. One of the main advantages of the Park equations is that all inductances are constant parameters. This advantage of the expanded Park theory does not exist.

\section{Identification of Park parameter}

In chapter 3, it is shown that the parameters of the Park equations cannot be derived by the phase-domain model. However, the Park equations are used as the standard analytic tool for calculating steady state operations and transients of the synchronous machines. Therefore, there is a high demand for Park parameters. But as shown, the Park parameters should not be derived by the geometric dimensions of synchronous machines. The classical procedure of deriving the parameters are measurements of the machine [8]. However, these methods often lead to inaccurate results. In [9] a more accurate method for the determination of Park parameters using optimization is presented and applied for transients during sub synchronous resonances.

In this article the parameters of the Park model are determined with the use of the evolution strategy by analyzing the three phase short circuit from no-load operation. The influence of saturation is neglected in this article but after the parameters are determined, the saturation can be regarded with different methods, e.g. described in [5]. 
The influence of the start parameters of the evolution strategy is under investigation in this article. First, there are no restrictions to the parameter: They are chosen randomly in the domain. In a second investigation the start parameters are predicted by the classical method described in [8]. Both investigations use the same settings for the evolution strategy.

\subsection{Evolution strategy}

The evolution strategy (ES) is a subset of the evolutionary algorithm that follows the natural biological evolution. The procedure of the ES can be separated into six steps:

1) defining the fitness function,

2) initializing starting population,

3) evaluating the actual fitness value,

4) creation of a new generation,

5) deterministic selection,

6) repeat steps 4) to 5) until termination.

The theory of the ES, especially of the creation of a new generation, is well known in literature, e.g. in [10-13]. In step 2 of the ES procedure, there are two options for initializing the starting population. The first option is to use random start parameters that are selected stochastically from the given domain and the second option is to predict the start parameters. For instance, the parameters are predicted by evaluating measurements or results of finite element analyses.

\subsection{Synchronous machine under investigation}

In this chapter the parameters of the round rotor synchronous machines of chapter 3 with linear magnetic material are determined. A finite element model is used to calculate the currents and the electromagnetic torque of a three phase short circuit from no-load operation. The resistance of the stator and the field windings can be measured easily. Since the Park model with one damper winding in the direct and in the quadrature axis with the additional inductance $L^{\prime}{ }_{c d}$ is chosen (Figs. 3 and 4), it is necessary to determine nine unknown parameters.

\subsection{Random start parameters}

The domain of the parameters is set to:

$$
\begin{gathered}
\left\{R_{x} \in \Re \mid-0.5 \mathrm{pu} \leq R_{x} \leq 0.5 \mathrm{pu}\right\}, \\
\left\{L_{x} \in \Re \mid-12 \mathrm{pu} \leq L_{x} \leq 12 \mathrm{pu}\right\} .
\end{gathered}
$$

Due to the fact, that the parameters of the Park equations cannot be determined from the phase-domain parameters, it should be valid that the parameters can be negative. In that case they are totally detached from physics.

All initial parameters are stochastic, randomly chosen in the whole domain. Creating the 1000 th new generation stops the ES. In every generation each population consists of 100 sets of parameters. 
The determined parameters are shown in the appendix. Fig. 9 shows the comparison of the finite element simulation and the result of the Park calculation with the optimized parameters. It is obvious that this set of parameters is not applicable. Even raising the number of creating new generations does not lead to a better result. Especially, the field current $I_{f}^{\prime}$ shows a large deviation between the finite element simulation and the analytic calculation.

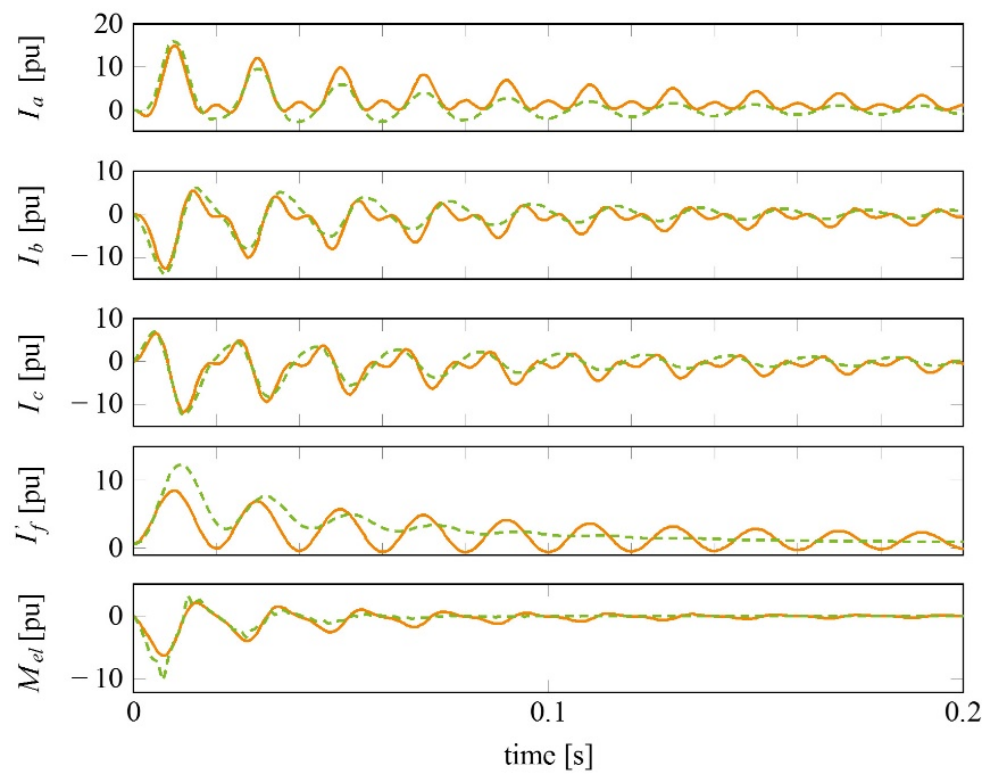

Fig. 9. Three phase short circuit: ES results with random start parameters

(FEA: green line, Park model: orange line)

\subsection{Predicted start parameters}

Before the evolution strategy to determine the parameters for the Park model is started, the initial parameters are predicted by evaluating the three phase short circuit with a method presented in [8]. This method leads to the parameters of just the direct axis. Therefore the quadrature axis parameters are set equal to the $d$-axis parameter. The rotor inductances $L^{\prime}{ }_{\text {of }}$ and $L^{\prime}{ }_{\sigma d}$ are functions of the Canay inductance $L^{\prime}{ }_{c d}$ [14], but in [8] there is no method to determine this inductance. So first the inductance $L^{\prime}{ }_{c d}$ is set to zero.

The settings of the ES are the same as in the investigation with random start parameters. Again creating the 1000th new generation stops the ES. In Fig. 10 it can be seen, that the optimized parameter set (see appendix) gives nearly perfect accordance with the results of the finite element analysis. There is only a small deviation of the field current $I_{f}^{\prime}$. The predicted start parameters vary widely from the optimized parameters. The result of the Park model with the start parameters, without the optimization, shows a huge deviation referred to the FE result. This results from the assumption of the same d- and q-axis parameters. Both models show good accordance to the FE results in steady state operations. It becomes obvious that the use of the ES determined adequate parameters. 

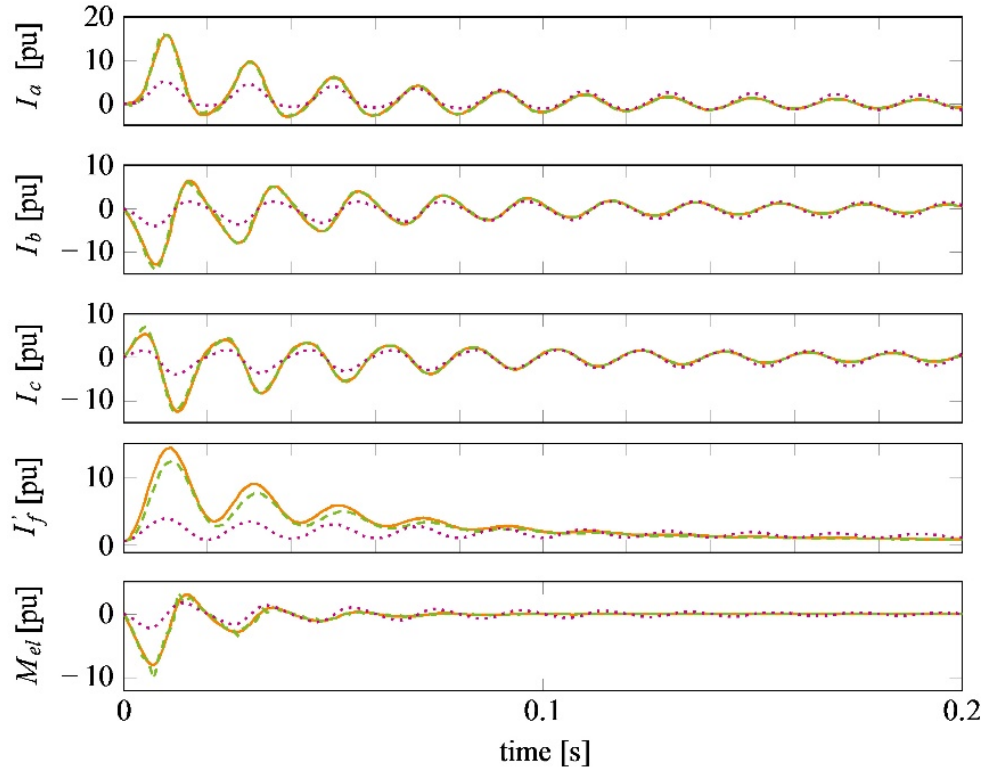

Fig. 10. Three phase short circuit: ES results with predicted start parameters (FEA: green line, Park model with optimized parameter: orange line, Park model with start parameter: purple line)

\subsection{Two phase short circuit}

Since the calculation with the optimized parameters with predicted start parameters shows a very good accordance to the FE results, only this parameter set is considered in this chapter.

The parameters of the synchronous machine were determined by an optimization method using the values of a three phase short circuit.

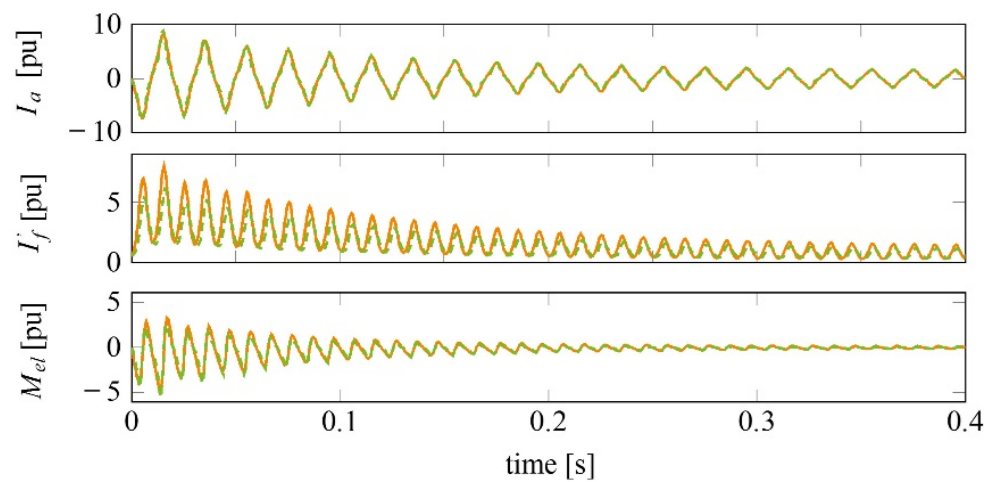

Fig. 11. Two phase short circuit: ES results with predicted start parameters

(FEA: orange line, Park model: green line)

It has to be proven that the parameters will be still correct during other operations of the synchronous machine. Therefore a two phase short circuit is calculated with the Park model and compared to a numerical calculation with FE (Fig. 11). Again, the analytic model values 
show a good accordance with the calculated FE values. In conclusion, the parameters fit for calculating transients of this synchronous machine.

\section{Conclusions}

In this article it is shown, that the derivation of the Park equations from the phase-domain model is not correct. The stator self and mutual inductances of two different types of synchronous machines are determined with the FE software Ansys Maxwell 2D. The simulation results show clearly, that the assumptions of the expanded Park transformation lead to an improper model. It is then reasonable to use the phase-domain model without any transformation because the major advantage of constant inductances in the Park equations is no longer fulfilled.

However, the Park theory is state of the art for analytic simulations of synchronous machines. Since, the parameters of the Park equations cannot be determined from the phase-domain model, a method is shown that identifies the needed parameters of the Park equations. This method is based on the evolution strategy and uses the currents and the electromagnetic torque of a three phase short circuit. The values can be measured directly or calculated with a finite element analysis. It is strongly recommend to predict the start parameters by evaluating a three phase short circuit. The calculation of a two phase short circuit shows, that the determined parameters are also valid for other transients.

\section{Appendix}

Optimized set of parameters I: determined with random start parameters [pu].

\begin{tabular}{ccccccc}
$R_{s}$ & $R_{f}^{\prime}$ & $R^{\prime}{ }_{d}$ & $R^{\prime}{ }_{q}$ & & & \\
\cline { 1 - 5 } 0.0095 & 0.0031 & 0.0309 & 0.3378 & & & \\
$L_{\sigma}$ & $L_{m d}$ & $L_{m q}$ & $L_{c d}^{\prime}$ & $L^{\prime}{ }_{\sigma f}$ & $L^{\prime}{ }_{\sigma d}$ & $L_{\sigma q}^{\prime}$ \\
\hline \hline 0.8365 & 2.6716 & 3.5523 & -3.253 & 2.3133 & 2.2610 & 2.1091
\end{tabular}

Optimized set of parameters II: determined with predicted start parameters [pu].

\begin{tabular}{ccccccc}
$R_{s}$ & $R_{f}^{\prime}$ & $R^{\prime}{ }_{d}$ & $R^{\prime}{ }_{q}$ & & & \\
\\
\hline \hline 0.0095 & 0.0031 & -0.0350 & 0.0562 & & & \\
$L_{\sigma}$ & $L_{m d}$ & $L_{m q}$ & $L^{\prime}{ }_{c d}$ & $L^{\prime}{ }_{\sigma f}$ & $L^{\prime}{ }_{\sigma d}$ & $L^{\prime}{ }_{\sigma q}$ \\
\hline \hline 0.0687 & 0.8302 & 0.8320 & 0.0082 & -0.0020 & -0.0090 & -0.0448
\end{tabular}

\section{References}

[1] Gajewski P., Pieńkowski K., Control of a Variable Speed Wind Turbine System with PMSG Generator, KOMEL, Maszyny elektryczne - Zeszyty Problemowe 107: 75-80 (2015).

[2] Schmuelling S., Kreischer C., Gołebiowski M., Comparison of Different Methods for Exciation of Synchronous Machines, KOMEL 107: 89-93 (2015).

[3] Park R., Two-reaction theory of synchronous machines generalized method of analysis-part I, Transactions of the American Institute of Electrical Engineers 48(3): 716-727 (1929). 
[4] Puchała A., Dynamika maszyn i układów elektromechanicznych, Państwowe Wydawnictwo Naukowe (1977).

[5] Krause P.C., Analysis of Electric Machinery, McGraw-Hill Book Company (1987).

[6] White D., Woodson H., Electromechanical Energy Conversion, [The M.I.T. core curriculum program in electrical engineering], John Wiley \& Sons (1959).

[7] Conradi A., Schmuelling C., Schmuelling S., Investigation on the effects of magnetic saturation in induction machines during transients, GSTF Journal of Engineering Technology (JET) 2(3) (2014).

[8] IEEE Guide: Test Procedures for Synchronous Machines Part I-Acceptance and Performance Testing Part II - Test Procedures and Parameter Determination for Dynamic Analysis, IEEE Std-115 (2010).

[9] Kreischer C., Kulig S., Göbel C., Applicability of Park transformation for the analysis of transient performance during subsynchronous resonances, Archives of Electrical Engineering 62(245): 401-415 (2013).

[10] Quagliarella D., Genetic algorithms and evolution strategy in engineering and computer science: recent advances and industrial applications, John Wiley \& Sons (1998).

[11] Beyer H., The Theory of Evolution Strategies, Springer (2001).

[12] Rechenberg I., Evolutionsstrategie: Optimierung technischer Systeme nach Prinzipien der biologischen Evolution, Frommann-Holzboog (1973).

[13] Pohlheim H., Evolutionäre Algorithmen: Verfahren, Operatoren und Hinweise für die Praxis, ser. VDI-Buch, Springer Berlin Heidelberg (1999).

[14] Canay M., Ersatzschemata der Synchronmaschine sowie Vorausberechnung der Kenngrössen mit Beispielen, PhD Thesis, Institut d'électrotechnique, Ecole polytechnique de l'Université de Lausanne (1968). 